\title{
A cluster-randomized field trial to reduce cesarean section rates with a multifaceted intervention in Shanghai, China
}

Lulu Zhang ${ }^{1 \dagger}$, Lin Zhang ${ }^{2 \dagger}$, Meng Li ${ }^{1,3+}$, Jie Xi ${ }^{4}$, Xiaohua Zhang ${ }^{5}$, Zhenni Meng ${ }^{6}$, Ying Wang ${ }^{7}$, Huaping Li ${ }^{8}$, Xiaohua Liu, ${ }^{6,9}$, Feihua Ju ${ }^{10}$, Yuping Lu ${ }^{11}$, Huijun Tang ${ }^{12}$, Xianju Qin ${ }^{13}$, Yanhong Ming ${ }^{1}$, Rong Huang ${ }^{1}$, Guohong Li $i^{3,14}$, Hongying Dai ${ }^{15}$, Rong Zhang ${ }^{16}$, Min Qin ${ }^{16^{*}}$, Liping Zhu ${ }^{16^{*}}$ and Jun Zhang ${ }^{1 *}$ (D

\begin{abstract}
Background: Cesarean section (CS) rate has risen dramatically and stayed at a very high level in China over the past two to three decades. Given the short- and long-term adverse effects of CS, effective strategies are needed to reduce unnecessary CS. We aimed to evaluate whether a multifaceted intervention would decrease the CS rate in China.

Methods: We carried out a cluster-randomized field trial with a multifaceted intervention in Shanghai, China, from 2015 to 2017. A total of 20 hospitals were randomly allocated into an intervention or a control group. The intervention consisted of more targeted health education to pregnant women, improved hospital CS policy, and training of midwives/doulas for 8 months. The study included a baseline survey, the intervention, and an evaluation survey. The primary outcome was the changes of overall CS rate from the pre-intervention to the post-intervention period. A subgroup analysis stratified by the Robson classification was also conducted to examine the CS change among women with various obstetric characteristics.

Results: A total of 10,752 deliveries were randomly selected from the pre-intervention period and 10,521 from the post-intervention period. The baseline CS rates were $42.5 \%$ and $41.5 \%$ in the intervention and control groups, respectively, while the post-intervention CS rates were $43.4 \%$ and $42.4 \%$, respectively. Compared with the control group, the intervention did not significantly reduce the $\mathrm{CS}$ rate (adjusted $\mathrm{OR}=0.92 ; 95 \% \mathrm{Cl} 0.73,1.15$ ). Similar results were obtained in subgroup analyses stratified by the risk level of pregnancy, maternal age, number of previous CS, or parity. Scarred uterus and maternal request remained the primary reasons for CS after the interventions in both groups. The intervention did not alter the perinatal outcomes (adjusted change of risk score $=-0.06 ; 95 \% \mathrm{Cl}-0.43,0.31$ ).

Conclusions: A multifaceted intervention including more targeted prenatal health education, improved hospital CS policy, and training of midwives/doulas, did not significantly reduce the CS rate in Shanghai, China. However, our experience in implementing a multifaceted intervention may provide useful information to other similar areas with high CS use.
\end{abstract}

Trial registration: This trial was registered at the Chinese Clinical Trial Registry (www.chictr.org.cn) (ChiCTR-IOR-16009041) on 17 August 2016.

Keywords: Cesarean section, Health education, Intervention, Midwives, Randomized controlled trial

\footnotetext{
*Correspondence: qinmin0902@163.com; shzhuliping@163.com; zhangjun@xinhuamed.com.cn

'Lulu Zhang, Lin Zhang and Meng Li contributed equally to this work and are considered as joint first authors.

${ }^{16}$ Shanghai Maternal and Child Health Center, Shanghai, China

'Ministry of Education-Shanghai Key Laboratory of Children's Environmental Health, Xinhua Hospital, Shanghai Jiao Tong University School of Medicine, Shanghai, China

Full list of author information is available at the end of the article
}

(c) The Author(s). 2020 Open Access This article is distributed under the terms of the Creative Commons Attribution 4.0 International License (http://creativecommons.org/licenses/by/4.0/), which permits unrestricted use, distribution, and reproduction in any medium, provided you give appropriate credit to the original author(s) and the source, provide a link to the Creative Commons license, and indicate if changes were made. The Creative Commons Public Domain Dedication waiver (http://creativecommons.org/publicdomain/zero/1.0/) applies to the data made available in this article, unless otherwise stated. 


\section{Introduction}

Cesarean section (CS) rate has increased dramatically in many parts of the world in the last few decades [1]. It was estimated that the average CS rate worldwide increased from $12.1 \%$ in 2000 to $21.1 \%$ in 2015 with an average annual rate of increase of $3.7 \%$ [1]. The CS rate in China began to rise in the early 1980s, with a sharp rise in the mid-1990s, and continued to rise from $28.8 \%$ in 2008 to $34.9 \%$ in 2014 [2, 3]. Reasons for the rapid rise in CS rate in China were multifactorial [4-6]. Among the commonly cited reasons are fear of labor pain, concerns about complications such as urinary incontinence and lower quality of sex life after vaginal delivery, misconception of CS being safer than vaginal delivery for the baby, poor experience of previous vaginal delivery, and auspicious dates [3, 4]. The shortage of nurses/midwives and the large volume of deliveries often lead to more convenient and scheduled CS. The constrained doctor-patient relationship and insufficient training in vaginal delivery also exacerbated the situation $[5,7]$. Higher financial incentives for CS versus vaginal delivery may lead to the preferred choice of CS $[2,4,5]$.

Extensive evidence has shown that CS without medical indications is associated with an increased risk of shortterm and long-term adverse outcomes as well as substantial economic burden [8-10]. The downside of widespread CS is now fully manifested in China where the government changed to a two-child policy recently. A high proportion of multiparous women have a scarred uterus, abnormal placenta implantation, and repeated CS $[11,12]$.

Shanghai is one of the largest cities in China. The CS rate increased from $17.5 \%$ in the early 1980 s to $55 \%$ in 2010 [13, 14]. Despite that the rate has declined to 47.9\% in 2016, it remains high [15]. Given that the causes of the high CS rate are multifactorial, previous studies suggested that multifaceted interventions be used to decrease the CS rate $[5,6,16-22]$. However, evidence concerning effective approaches to reduce unnecessary CS is limited [23, 24], especially in China and other lowand mid-income countries. Therefore, we conducted a cluster-randomized field trial in Shanghai, China, where the CS rate is very high $[2,17,25]$, to examine the effects of a multifaceted strategy targeting mothers, health professionals, and hospital policy to reduce the CS rate.

\section{Methods}

\section{Study design and hospitals}

This stratified, cluster-randomized, parallel-group field trial was conducted to examine the effects of a multifaceted intervention on the use of CS in 20 hospitals in Shanghai, China, from 2015 to 2017. Hospitals were invited to participate in this trial and were informed that they could be assigned to either intervention or control group. Seven tertiary hospitals and 13 secondary hospitals agreed to participate. Primary care hospitals were not included in the present study, as they usually do not provide obstetric services, and very few women are delivered outside of the hospital. The participating hospitals delivered approximately half of all births in Shanghai (approximately 200,000 births per year).

An ethical approval was obtained from the Ethics Review Board of the Xinhua hospital, Shanghai Jiao Tong University School of Medicine and other participating hospitals (Approval number: XHEC-C-2016-095).

\section{Randomization and masking}

Hospitals were first stratified by their levels (tertiary vs secondary). Within each stratum, hospitals were randomly assigned to the intervention and control groups and designated as the units of randomization to ensure that there would be minimal cross contamination between the intervention and control groups. The randomization was conducted by the data management group. The study included a 6-month pre-intervention (baseline survey) period, an 8-month intervention period, and a 6-month post-intervention (evaluation survey) period. No masking was applied in this study.

\section{Baseline survey}

Before the intervention, we conducted a baseline survey. A total of 62,653 births were delivered in the 20 hospitals from January 1 to June 30 in 2016. A random sample of all births was selected. To ensure the precision of the CS rate estimates, we randomly selected $20 \%$ of the total births in hospitals with an annual delivery volume under 10,000 , and $10 \%$ in hospitals with an annual delivery volume over 10,000 [26]. On average, about 500 records were extracted per hospital. To make our findings comparable to other studies, we further restricted the analysis to women whose newborns had a gestational age of at least 24 weeks or weighed at least $500 \mathrm{~g}$ at delivery. Finally, a total of 10,807 deliveries remained to represent the total births in these hospitals during that period. Medical records of mothers and newborns were retrieved, and information on maternal demographic characteristics, reproductive history, and maternal and neonatal conditions were abstracted by specially trained research staff for both the baseline and evaluation surveys.

\section{Interventions}

A multifaceted intervention was developed based on previous research $[5,22,27]$. It consisted of three components. First, a targeted health education program on top of the regular prenatal education was developed to familiarize pregnant women with the process of natural childbirth, and the health benefits and risks of CS. 
Educational brochures, 15 online and offline courses, and free outpatient consultations were offered to women free of charge in the intervention hospitals. These education programs covered the whole pregnancy from booking to delivery. A set of brochures on various topics of CS and natural birth were developed. A number of prenatal health courses were recorded and placed online for women to view at any time. Some talks by health professionals were broadcasted live. In-person classes were also held at weekends for women to attend free of charge. The list of courses was described in more detail in Additional file 1.

Second, after a careful review of the literature, a focus group discussion with obstetricians, midwives, and hospital administers, and consultation with hospital management, an improved hospital CS policy was established and promoted in the intervention hospitals. The policy included three measures: To install a CS second opinion process, i.e., if an obstetrician decides to perform a CS on a woman, he/she needs to request a review by the unit chief or a designated senior physician for a second opinion [16, 27, 28]. The obstetric departments in the intervention hospitals were also encouraged to conduct regular peer reviews of CS indications, post the monthly CS rate, and implement a reward system [16, 27-31].

Third, several training courses with a specially designed syllabus were offered to midwives and doulas in the intervention hospital to improve their skills.

The intervention was implemented from September 1, 2016, to April 30, 2017. The control group did not receive any of the above interventions except for providing the usual care.

\section{Evaluation survey}

A total of 54,257 births were delivered in the participating hospitals during the evaluation period from May 1 to October 31 in 2017. A random sample was selected, and data were abstracted in the same way as that of the baseline survey. A total of 10,553 deliveries remained for analyses (Additional files 2, 3, and 4).

\section{Assessment of outcomes}

The main outcomes were at the individual participant level within hospitals (randomization units). The primary outcome was the changes of the overall CS rate from the pre-intervention to the post-intervention period. A subgroup analysis stratified by the Robson classification was also conducted to examine the CS change among women with various obstetric characteristics. The secondary outcomes were gestational weight gain (GWG), obstetrical interventions, and perinatal outcomes. GWG was defined as the difference between documented weight at the first and last prenatal visit just before delivery. Obstetrical interventions included planned and intrapartum CS rate, artificial rupture of the membranes, labor induction, oxytocin use during labor, epidural analgesia, use of doula, assisted vaginal delivery, and episiotomy. Planned and intrapartum CS were defined as CS before and after the onset of labor, respectively. Labor induction was defined as artificially induced uterine contraction. Perinatal outcomes were measured by a composite score, defined by the American College of Obstetricians and Gynecologists (ACOG) Quality Improvement and Patient Safety Committee (QuIPS) [32]. Each of the 10 outcomes was assigned a weighted score indicating the severity: maternal death, 750 points; intrapartum or neonatal death $>2500 \mathrm{~g}, 400$ points; uterine rupture, 100 points; maternal admission to ICU, 65 points; birth trauma, 60 points; return to operating room/labor and delivery, 40 points; admission to NICU $>2500 \mathrm{~g}$ and for $>24 \mathrm{~h}, 35$ points; APGAR $<7$ at $5 \mathrm{~min}$, 25 points; blood transfusion, 20 points; and $3^{\circ}$ - or $4^{\circ}$ perineal tear, 5 points. The individual perinatal risk score was computed by the sum of the scores of all the 10 outcomes (if any) to manifest perinatal outcome for each mother.

\section{Assessment of covariates}

Maternal age was treated as a continuous variable. Information on maternal height, weight at delivery, nulliparous (yes/no), assisted reproductive technology (ART; yes/ no), previous cesarean delivery (yes/no), gestational age (GA) at delivery, birthweight of newborn, pathology (yes/no), and hospital level (tertiary hospitals/secondary hospitals) were collected. Body mass index (BMI) was calculated as maternal weight at delivery in kilograms divided by the square of height in meters $\left(\mathrm{kg} / \mathrm{m}^{2}\right)$.

A pregnancy was considered as morbid if any of the following conditions was met: non-cephalic presentation of the fetus, placental abruption, placenta previa, uterine rupture, hypertensive disorders of pregnancy (including gestational hypertension, preeclampsia, eclampsia, and HELLP syndrome), heart disease, deep venous thrombosis, kidney disease, pre-gestational and gestational diabetes mellitus, pre-gestational and gestational thyroid disease (including hyperthyroidism, hypothyroidism and others), premature rupture of membranes (gestational age < 37 weeks), $\mathrm{Rh}$ incompatibility, or congenital malformation.

A pregnancy was considered as low-risk if the newborn was born in cephalic presentation, and the mothers were aged 18 or above and younger than 40 years old, gave a term birth (37-41 completed weeks of gestation), had a pre-pregnancy BMI between 17 and $28 \mathrm{~kg} / \mathrm{m}^{2}$, and were without previous ART, CS, prior or current stillbirth, and morbidity during pregnancy defined above. In contrast, a pregnancy was considered as high-risk with any of the above conditions. 


\section{Statistical analysis}

Based on the overall CS rate of $45 \%$ in Shanghai, we estimated that the intervention may reduce the CS rate by 7 to $38 \%$. Assuming that the unit size was about 500 subjects per hospital and the intraclass correlation coefficient was 0.011 , we calculated that we would need to enroll 20 hospitals for the purpose of the study to have $90 \%$ power to detect $7 \%$ reduction in CS rate. A twosided alpha significance level of 0.05 was used.

Continuous variables were described as mean (standard deviation), whereas categorical variables were presented as numbers and percentages. An intention-totreat analysis was applied according to the assignment of randomization at the beginning of the study. Given the clustering of women (final analysis units) within hospitals (randomization units), generalized estimating equations (GEE) were used to assess the effects of the multifaceted intervention on CS rates, GWG, obstetrical interventions, and perinatal outcomes, separately, adjusting for maternal age, BMI at delivery, parity, ART, previous CS history, GA at delivery, birthweight of the newborn, pregnancy complications, and hospital level. Adjusted OR, adjusted $\beta$, and corresponding 95\% CIs were computed to compare the changes between the intervention group and the control group from the preto the post-intervention period. For the GEE models that did not converge, a logistic regression model was used with $p$ values of less than 0.001 being considered as statistically significant and $p$ values of less than 0.003 being marginally significant $[33,34]$.

We conducted stratified analyses by the risk level of the mothers (low vs. high), maternal age ( $\geq 35$ vs. $<35$ ), the number of previous CS ( 0 vs. $\geq 1)$, and parity (primipara vs. multipara without a previous CS vs. multipara with a previous CS or not). Additionally, to identify factors that were negatively associated with CS, we evaluated the intervention effect in each group of mothers by the modified Robson Classification System [35]. Five basic obstetric characteristics were used by the modified Robson classification system to categorize all subjects admitted for delivery: parity (nulliparous, multiparous with or without a previous CS), onset of labor (spontaneous labor, induced labor, or CS before labor), gestational age (preterm birth or full term), fetal presentation (cephalic, breech, transverse or oblique lie), and number of fetus (singleton or multiplets). In order not to miss significant information for the success of induction and its contribution to the CS rate, the modified Robson classification divided induced labor and CS before labor into two groups for nulliparous and multiparous women, respectively (groups 2, 3, 5, and 6). On the other hand, the number of women with transverse or oblique fetal lie was small but the CS rates for non-cephalic presentations were very high. Thus, these groups were combined into one (group 8). Moreover, subjects who lacked at least one of the above five obstetric characteristics were placed in the unknown group (group 99). After the appropriate expansion and reduction in certain categories, the total number of groups remained at 10, plus the unknown group. Specifically, group 1 (nulliparous, spontaneous: abbreviated as NS): nulliparous women with singleton cephalic pregnancy, $\geq 37$ weeks in spontaneous labor; group 2 (nulliparous, induced: $\mathrm{NI}$ ): nulliparous women with singleton cephalic pregnancy, $\geq 37$ weeks in induced labor; group 3 (nulliparous, cesarean: NC): nulliparous women with singleton cephalic pregnancy, $\geq 37$ weeks who were delivered by CS before labor; group 4 (multiparous, spontaneous: MS): multiparous women without a previous CS, with singleton cephalic pregnancy, $\geq 37$ weeks in spontaneous labor; group 5 (multiparous, induced: MI): multiparous women without a previous CS, with singleton cephalic pregnancy, $\geq 37$ weeks in induced labor; group 6 (multiparous, cesarean: $\mathrm{MC}$ ): multiparous women without a previous CS, with singleton cephalic pregnancy, $\geq 37$ weeks who were delivered by CS before labor; group 7 (previous cesarean: PC): multiparous women with a previous CS, with singleton cephalic pregnancy, $\geq 37$ weeks; group 8 (breech: BR): all women with a singleton pregnancy with a breech, transverse, or oblique lie; group 9 (twin: TW): all women with multiple pregnancies (twins or higherorder multiples); group 10 (preterm: PT): all women with a singleton cephalic pregnancy, $<37$ weeks [35].

All statistical analyses were conducted with SAS 9.4 software (SAS Institute Inc., Cary, NC). This trial was registered at the Chinese Clinical Trial Registry (www. chictr.org.cn) (ChiCTR-IOR-16009041).

\section{Results}

The intervention group consisted of three tertiary and seven secondary hospitals, and the control group was composed of four tertiary and six secondary hospitals. From January 1, 2016, to October 31, 2017, a total of 21, 360 deliveries were randomly sampled during the study period, including 10,807 deliveries in the preintervention and 10,553 in the post-intervention period (Fig. 1). After excluding women with missing information on labor and delivery, 21,273 women (99.6\%) were included in the final analysis. Baseline characteristics including maternal age, parity, GA at delivery, CS history, risk level of the pregnancy, birth outcome, and neonatal birthweight were generally similar between the intervention and control groups. In contrast, newborns were slightly more likely to be non-cephalic presentation in the intervention group than in the control group (5.3\% vs. $4.3 \%$ ) (Table 1 ).

The baseline CS rates were $42.5 \%$ and $41.5 \%$ in the intervention and control groups, respectively, versus 


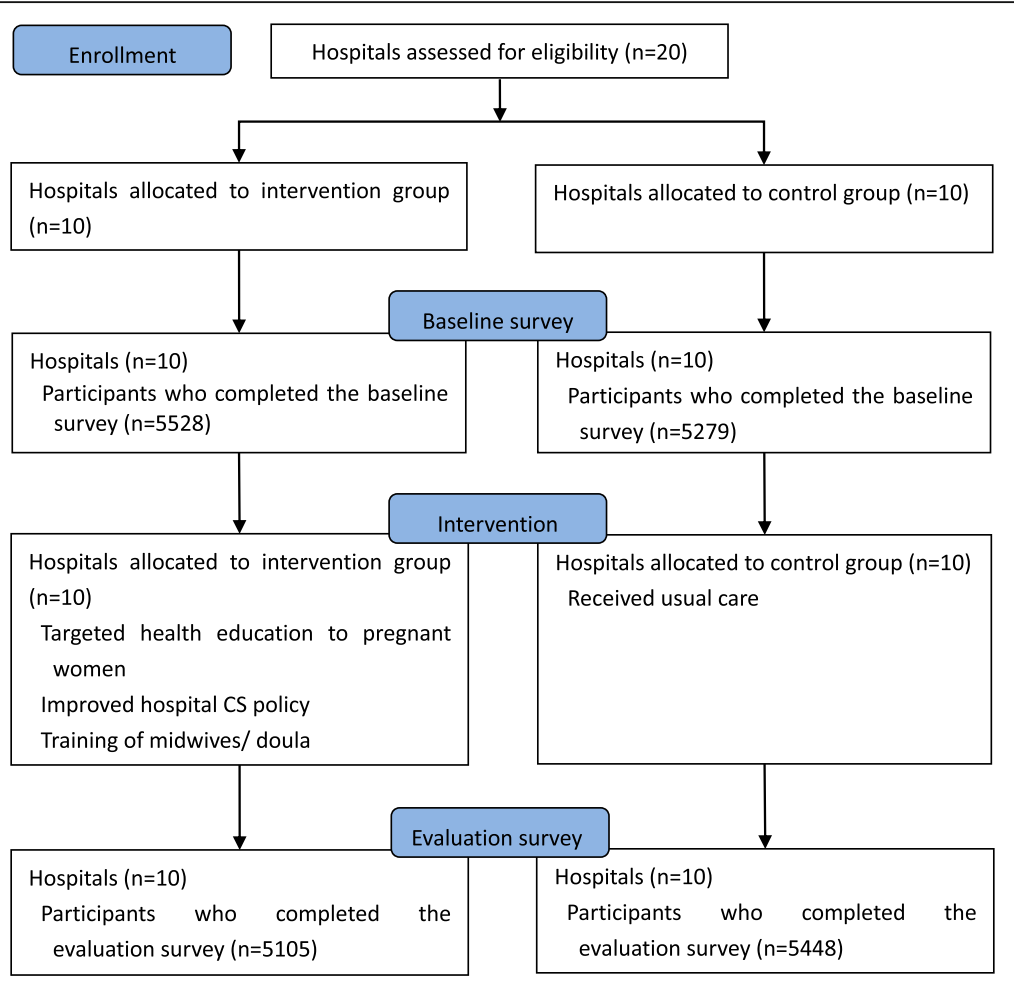

Fig. 1 Trial profile

$43.4 \%$ and $42.4 \%$ in the post-intervention period, respectively. The intervention did not significantly reduce the CS rate when comparing with the control group during the same period (adjusted $\mathrm{OR}=0.92 ; 95 \% \mathrm{CI} 0.73$, $1.15 ; p=0.44$ ) (Table 2). Similar results were obtained after we stratified the women by the risk level of the pregnancy, maternal age, number of previous CS, or parity. Among the low-risk women, there was a small reduction in CS rate in both groups from the baseline to the post-intervention periods $(-3.6 \%$ and $-2.8 \%$, respectively). In contrast, the CS rates in both groups increased during the same period in high-risk pregnancies ( $2.5 \%$ and $4.0 \%$, respectively). Although none of the subgroup analyses showed any statistically significant reduction, all the point estimates of the adjusted odds ratios were below 1 .

Table 3 shows the CS rate by the categories of the modified Robson Classification System and by group allocation and period. Similarly, the intervention did not significantly affect the CS rates across the Robson categories.

The baseline GWG were 13.2 (SD 5.3) $\mathrm{kg}$ and 12.3 (SD 5.5) $\mathrm{kg}$ in the intervention and control groups, respectively, versus 11.2 (SD 4.8) $\mathrm{kg}$ and 11.2 (SD 5.1) kg in the post-intervention period, respectively. The intervention did not significantly reduce the GWG when comparing with the control group during the same period (adjusted $\beta=-0.05 ; 95 \% \mathrm{CI}-0.11,0.01 ; p=0.11$ ).
Scarred uterus, CS by maternal request without medical indication, abnormal fetal heart rate pattern, breech or transverse presentation, prolonged labor, and macrosomia ranked the top six primary indications for CS in both groups during the baseline and the evaluation periods. The proportion of CS due to scarred uterus had increased from the baseline to the evaluation period in both groups ( $4.6 \%$ and $3.6 \%$, respectively) (Table 4$)$. The frequencies of obstetrical interventions were similar between the two groups before and after the intervention (Table 5). However, among women who had a trial of labor, the intervention appeared to have had a suggestive but statistically non-significant effect on assisted vaginal delivery $(\mathrm{OR}=0.61,95 \% \mathrm{CI} 0.31,1.21)$ (Table 5).

Few women had severe complications. The proportion of women with an individual perinatal risk score defined by ACOG QuIPS above zero remained virtually the same from the baseline to the evaluation period in both groups $(-0.3 \%$ vs. $-0.2 \%$, respectively).

\section{Discussion}

This trial showed that a multifaceted intervention did not reduce the high CS rate in Shanghai, China. Scarred uterus and maternal request were still the primary indications for CS even after the intervention. To our best knowledge, our study was so far the first randomized trial to reduce the CS rate by a multifaceted intervention in China. 
Table 1 Characteristics of hospitals and patients by group allocation during the baseline period, Shanghai, China

\begin{tabular}{|c|c|c|}
\hline & $\begin{array}{l}\text { Intervention Group } \\
(N=5498)\end{array}$ & $\begin{array}{l}\text { Control grou } \\
(N=5254)\end{array}$ \\
\hline \multicolumn{3}{|l|}{ Hospitals } \\
\hline \multicolumn{3}{|l|}{ Type of hospital, no. (\%) } \\
\hline Tertiary hospitals & $1941(35.3)$ & $1813(34.5)$ \\
\hline Secondary hospitals & $3557(64.7)$ & $3441(65.5)$ \\
\hline \multicolumn{3}{|l|}{ Patients } \\
\hline \multicolumn{3}{|l|}{ Maternal age at delivery, years } \\
\hline < 18 years, no. (\%) & $12(0.2)$ & $19(0.4)$ \\
\hline 18-34 years, no. (\%) & $4842(89.9)$ & $4686(90.1)$ \\
\hline$\geq 35$ years, no. (\%) & $533(9.9)$ & $494(9.5)$ \\
\hline Missing & 111 & 55 \\
\hline $\begin{array}{l}\text { Pre-pregnancy body mass } \\
\text { index, } \mathrm{kg} / \mathrm{m}^{2}\end{array}$ & $22.0 \pm 3.2$ & $22.3 \pm 3.3$ \\
\hline \multicolumn{3}{|l|}{ Parity, no. (\%) } \\
\hline 0 & $3546(64.5)$ & $3399(64.7)$ \\
\hline 1 & $1828(33.3)$ & $1715(32.7)$ \\
\hline$\geq 2$ & $121(2.2)$ & $137(2.6)$ \\
\hline Missing & 3 & 3 \\
\hline \multicolumn{3}{|l|}{ Gestational age at delivery, no. (\%) } \\
\hline$<37$ weeks & $312(5.7)$ & $292(5.6)$ \\
\hline $37-41$ weeks & $5167(94.0)$ & $4948(94.2)$ \\
\hline$\geq 42$ weeks & $19(0.4)$ & $14(0.3)$ \\
\hline \multicolumn{3}{|l|}{ Previous cesarean deliveries, no. (\%) } \\
\hline No & $4722(86.0)$ & $4516(86.1)$ \\
\hline Yes & $768(14.0)$ & $727(13.9)$ \\
\hline Missing & 8 & 11 \\
\hline \multicolumn{3}{|l|}{ Risk level of pregnancy, no. (\%) } \\
\hline Low & $2830(51.5)$ & $2671(50.8)$ \\
\hline High & $2668(48.5)$ & $2583(49.2)$ \\
\hline \multicolumn{3}{|l|}{ Presentation of the baby } \\
\hline Cephalic & $5088(94.7)$ & $4964(95.7)$ \\
\hline Breech/transverse/oblique lie & $284(5.3)$ & $222(4.3)$ \\
\hline Missing & 126 & 68 \\
\hline \multicolumn{3}{|l|}{ Mode of delivery } \\
\hline Vaginal delivery & $3034(55.2)$ & $3003(57.2)$ \\
\hline Assisted vaginal delivery & $128(2.3)$ & $70(1.3)$ \\
\hline Planned cesarean delivery & $2000(36.4)$ & $1855(35.3)$ \\
\hline $\begin{array}{l}\text { Intrapartum cesarean } \\
\text { delivery }\end{array}$ & $336(6.1)$ & $326(6.2)$ \\
\hline \multicolumn{3}{|l|}{ Birth outcome } \\
\hline Live birth & 5479 (99.7) & $5236(99.7)$ \\
\hline Fetal death & $14(0.3)$ & $13(0.3)$ \\
\hline Stillbirth & $1(0.02)$ & $2(0.04)$ \\
\hline Neonatal death & $4(0.07)$ & $3(0.06)$ \\
\hline
\end{tabular}

Table 1 Characteristics of hospitals and patients by group allocation during the baseline period, Shanghai, China (Continued)

\begin{tabular}{lll}
\hline & $\begin{array}{l}\text { Intervention Group } \\
(N=5498)\end{array}$ & $\begin{array}{l}\text { Control group } \\
(N=5254)\end{array}$ \\
\hline $\begin{array}{l}\text { Neonatal birthweight, g } \\
<1500, \text { no. (\%) }\end{array}$ & $16(0.3)$ & $17(0.3)$ \\
1500-2499, no. (\%) & $185(3.4)$ & $191(3.6)$ \\
2500-3999, no. (\%) & $4901(89.2)$ & $4663(88.8)$ \\
$\geq 4000$, no. (\%) & $395(7.2)$ & $383(7.3)$ \\
$\quad$ Missing & 1 & 0 \\
\hline Outcome: twins combined & \\
Neonatal admission to ICU: twins combined &
\end{tabular}

Numerous attempts have been made to reduce the CS rate around the world [27, 28, 34, 36-38]. Unfortunately, the impact of a single intervention approach has been inconsistent and mostly limited [39]. Chaillet et al. used multifaceted interventions, including audits of indications for CS, provision of feedback to health professionals, and implementation of best practices, and reported a statistically significant but small reduction in the CS rate (adjusted risk difference $=-1.8 \% ; 95 \% \mathrm{CI}-3.8 \%,-0.2 \%)$ [34]. A trial by Althabe et al. showed that a hospital policy of mandatory second opinion had a similar statistically significance but marginal reduction in CS use (adjusted risk difference $=-1.9 \%$; 95\% CI $-3.8 \%,-0.1 \%$ ) [27] .

On the other hand, two Chinese retrospective observational studies suggested that multifaceted interventions involving government policy, finical incentives, local benchmarking, health education for health professionals and pregnant women, doula care, and access to labor analgesia could decrease the CS use effectively [40, 41]. In a retrospective study at a large maternity hospital in Shanghai, Liu et al. compared CS rates before and after the implementation of a multifaceted intervention [41], which included government and hospital measures. The government measure consisted of fixing per-patient reimbursement by the government health insurance regardless of the delivery mode, and ranking the obstetric departments by the CS rate. The hospital measure included free perinatal healthcare classes, improving women's childbirth experience by allowing family and an experienced midwife to stay with them during labor and offering labor analgesia, ranking physicians' performance within the hospital by CS rate. After the interventions, there was a $31 \%$ reduction in the CS rate, with an OR of 0.69 [95\% CI 0.66-0.71]. However, the study did not separate the effects of the government and hospital measures.

$\mathrm{Yu}$ et al. conducted a retrospective pre-/post-intervention study which focused on CS on maternal request 


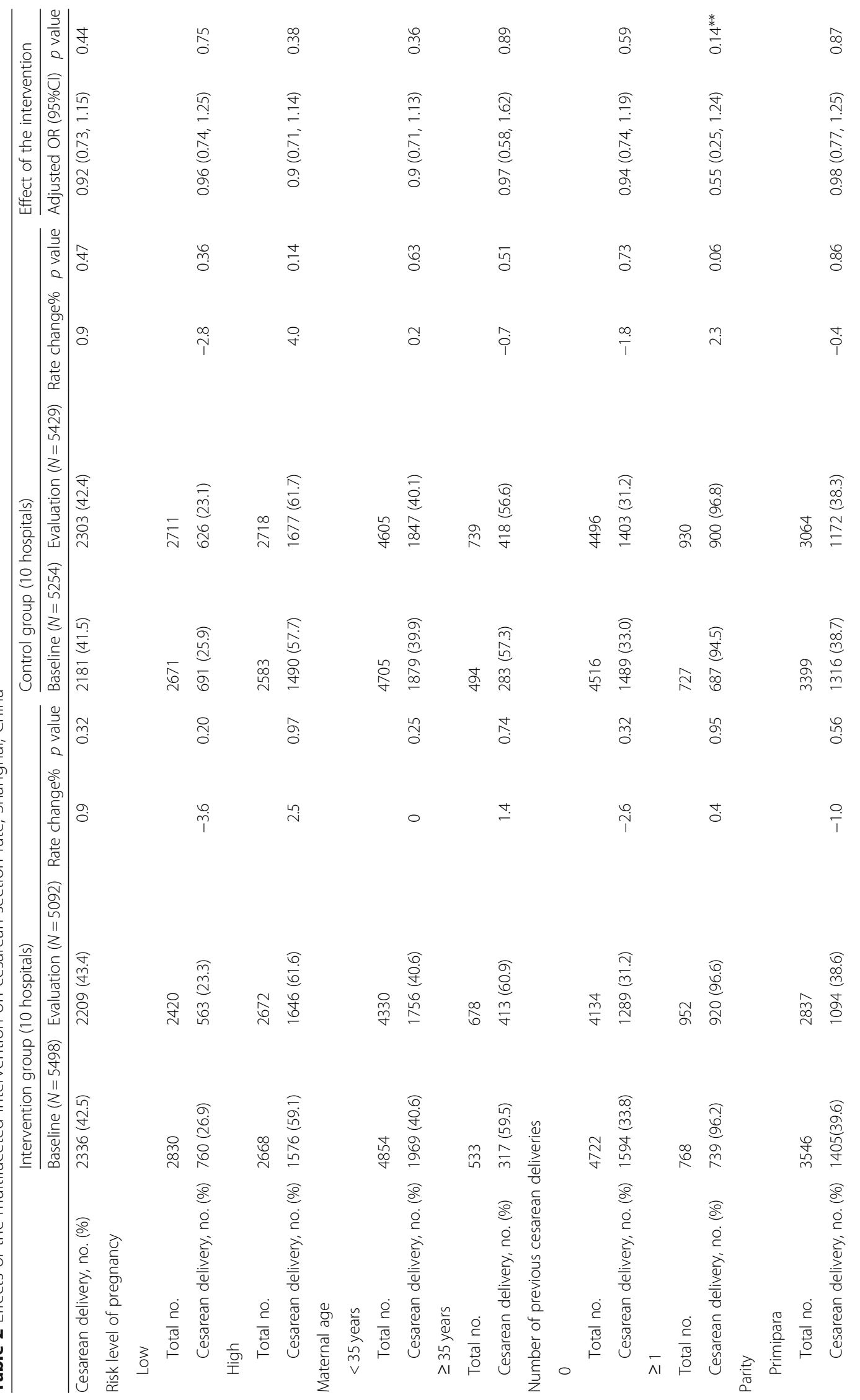




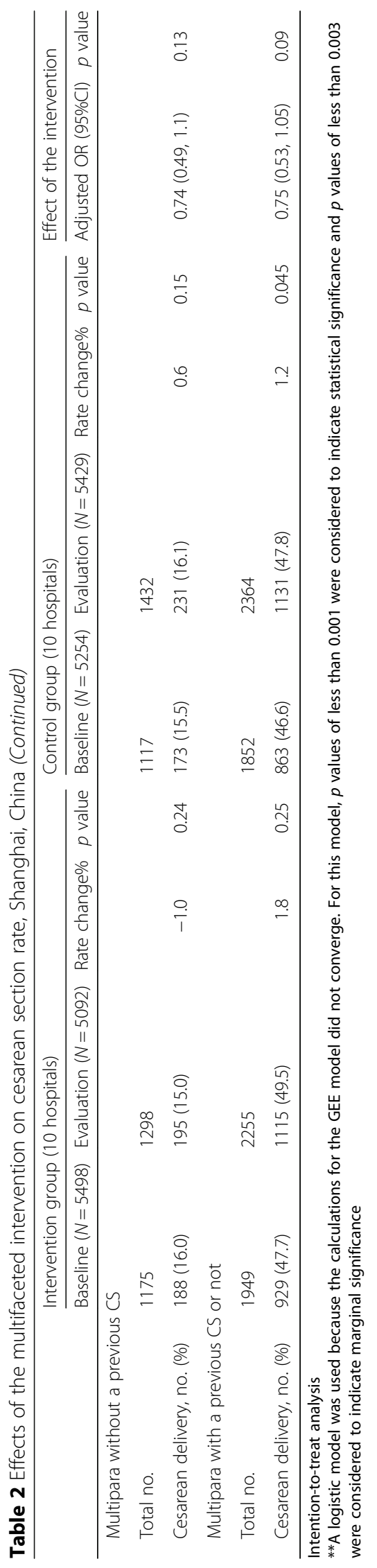


Zhang et al. BMC Medicine $\quad$ (2020) 18:27

Page 9 of 15

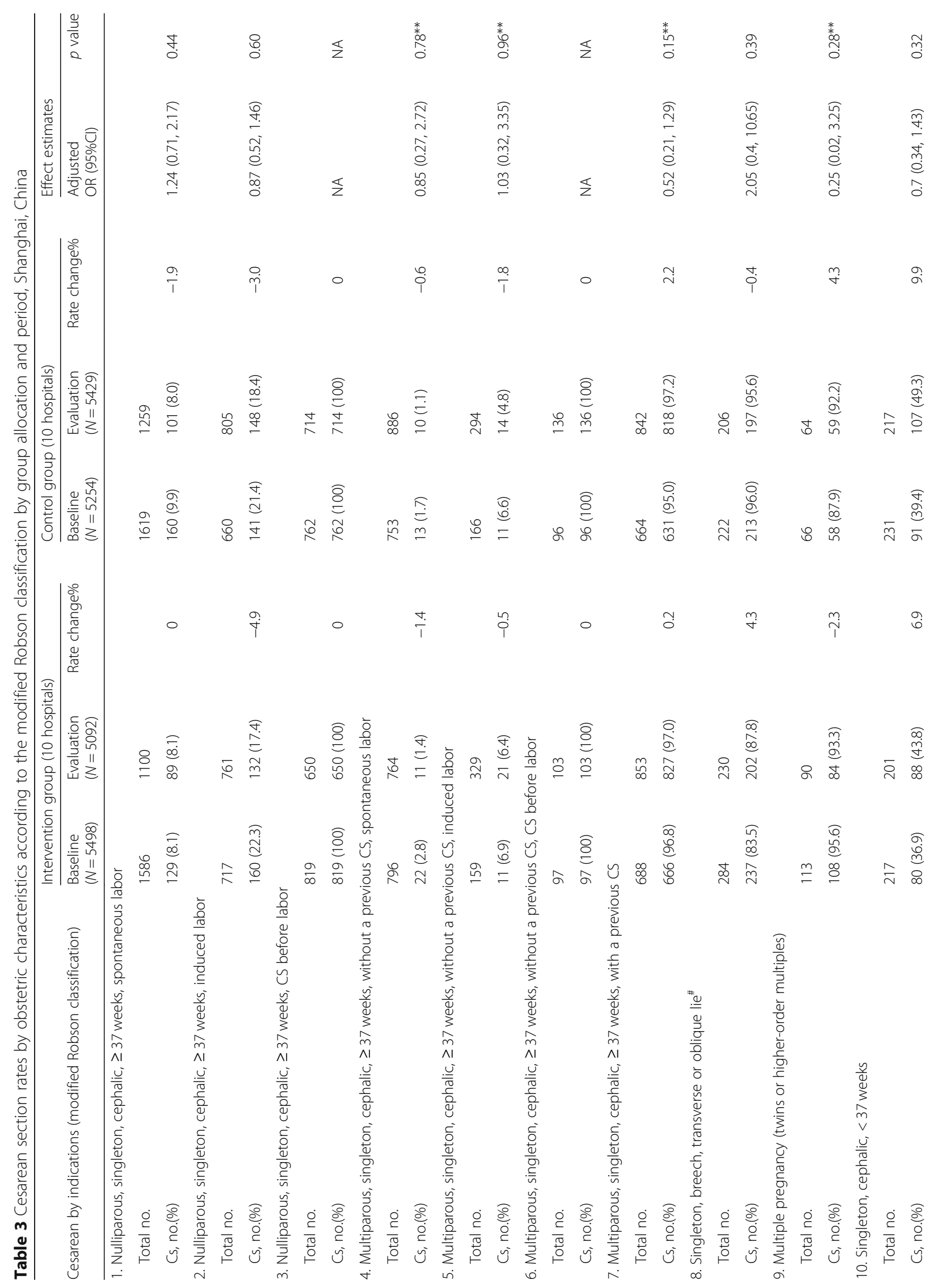




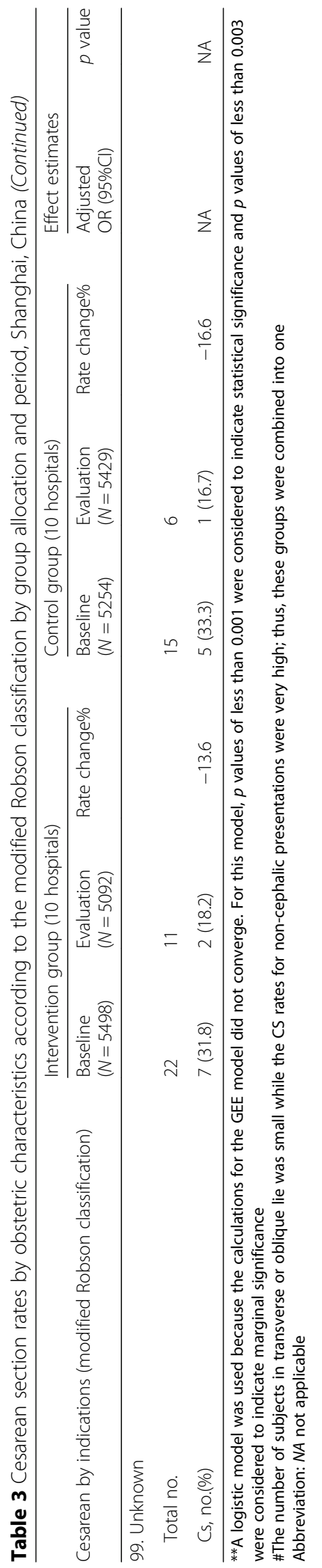


Table 4 Hospital-based cesarean section rates by the primary indication and by the group allocation during the baseline and postintervention periods, Shanghai, China

\begin{tabular}{|c|c|c|c|c|c|}
\hline \multirow[b]{2}{*}{ Cesarean indications } & \multicolumn{2}{|c|}{ Intervention group (10 hospitals) } & \multicolumn{2}{|c|}{ Control group (10 hospitals) } & \multirow[t]{2}{*}{$p$ value } \\
\hline & Baseline $(N=5498)$ & Evaluation $(N=5092)$ & Baseline $(N=5254)$ & Evaluation $(N=5429)$ & \\
\hline Scarred uterus, no. (\%) & $716(13.0)$ & $897(17.6)$ & $632(12.3)$ & $863(15.9)$ & 0.24 \\
\hline Cesarean section without medical indication, no. (\%) & $480(8.7)$ & $401(7.9)$ & $456(8.7)$ & $429(7.9)$ & 0.21 \\
\hline Abnormal fetal heart rate (FHR) pattern, no. (\%) & $325(5.9)$ & $257(5.1)$ & $413(7.9)$ & $395(7.3)$ & 0.08 \\
\hline Breech or transverse presentation, no. (\%) & $230(4.2)$ & $173(3.4)$ & $200(3.8)$ & $178(3.3)$ & 0.24 \\
\hline Macrosomia, no. (\%) & $135(2.5)$ & $106(2.1)$ & $109(2.1)$ & $92(1.7)$ & 0.71 \\
\hline Prolonged labor, no. (\%) & $65(1.2)$ & $69(1.4)$ & $49(0.9)$ & $39(0.7)$ & 0.30 \\
\hline Other indications, no. (\%) & $382(7.0)$ & $303(6.0)$ & $317(6.0)$ & $300(5.5)$ & 0.11 \\
\hline Total, no. (\%) & $2333(42.4)$ & $2206(43.3)$ & $2176(41.4)$ & $2296(42.3)$ & 0.01 \\
\hline
\end{tabular}

changed by institutional interventions and government policy [40]. Institutional interventions consisted of three aspects: providing health education to mothers and their families; training obstetricians and midwives, issuing CS indications and guidelines, and conducting audits every month; promoting labor analgesia and doula care by midwives. These interventions were similar to ours. In addition, the central and local government policy directly addressed the financial and management aspects. The overall CS rate declined by $1.3 \%$ and $8.3 \%$ attributable to the institutional and government interventions, respectively. Nonetheless, the previous studies in China were retrospective observational data analyses. The true impact needs to be evaluated in a randomized controlled fashion.

Despite that these studies were conducted in quite different settings and cultures, and all of them showed some effects of the multifaceted interventions, they seemed to have some common characteristics. First, studies with interventions initiated by academic organizations reduced CS rate only to a modest degree [24, 27, 34]. Instead, the interventions initiated by the maternity hospitals themselves showed a larger reduction in CS use [36, 42, 43]. Furthermore, the government-led efforts were more effective when the CS rate was included as a benchmark for hospital performance [40, 41]. For example, a nationwide intervention strategy in Portugal even reversed the national upward trend of the CS rate [38]. It was worth noting that the two studies in China evaluated the changes of the CS rate around 2012, when WHO published a report on the high CS rate, especially for those without medical indications in China. After the WHO report, the Chinese government became increasingly concerned of the adverse health impacts of the high CS rate and took a series of measures to address this issue [44].

It is also understandable that interventions conducted in a single hospital tended to gain more support from medical opinion leaders and, thus, easier to form a concerted action in the hospital. Similarly, the governmentled interventions could be directly implemented in healthcare systems. In contrast, interventions initiated by academic researchers did not have such advantages. Kingdon et al. and Chaillet et al. found that the negotiation of health professionals with healthcare system and the practice environment including unit leadership, policy, availability of equipment, and the extent to undertake the guideline recommendation were the major keys to decrease the CS rate successfully $[22,45]$.

Due to a high CS rate for over a decade, a higher proportion of women in China have a scarred uterus comparing to other countries. Women with a previous CS were more likely to select repeat CS. This explains why our CS rate actually increased from the baseline to the post-invention period because during our trial, the twochild policy was instituted. The proportion of multiparas increased, many of whom had a previous CS, leading to a higher overall CS rate. However, in nulliparas, the CS rates did decline but the difference between the intervention and control groups did not reach statistical significance. It should be noted that the considerable flux of declining primary CS and increasing repeat CS may hamper a substantial decline in the overall CS rate in the near future.

Our study has several limitations. First, although we provided women more targeted health education via the online and offline programs free of charge in the intervention hospitals, approximately half of the women in the intervention hospitals participated in our targeted health education program. Thus, the overall impact may have been diluted. Second, due to a high volume of deliveries, it is challenging to provide one-on-one doula support throughout labor in most hospitals. A total of $30-40 \%$ had doula support, among whom some women shared a doula [46]. Third, despite our effort in promoting the improved hospital CS policy, the degree of adaptation varied by 


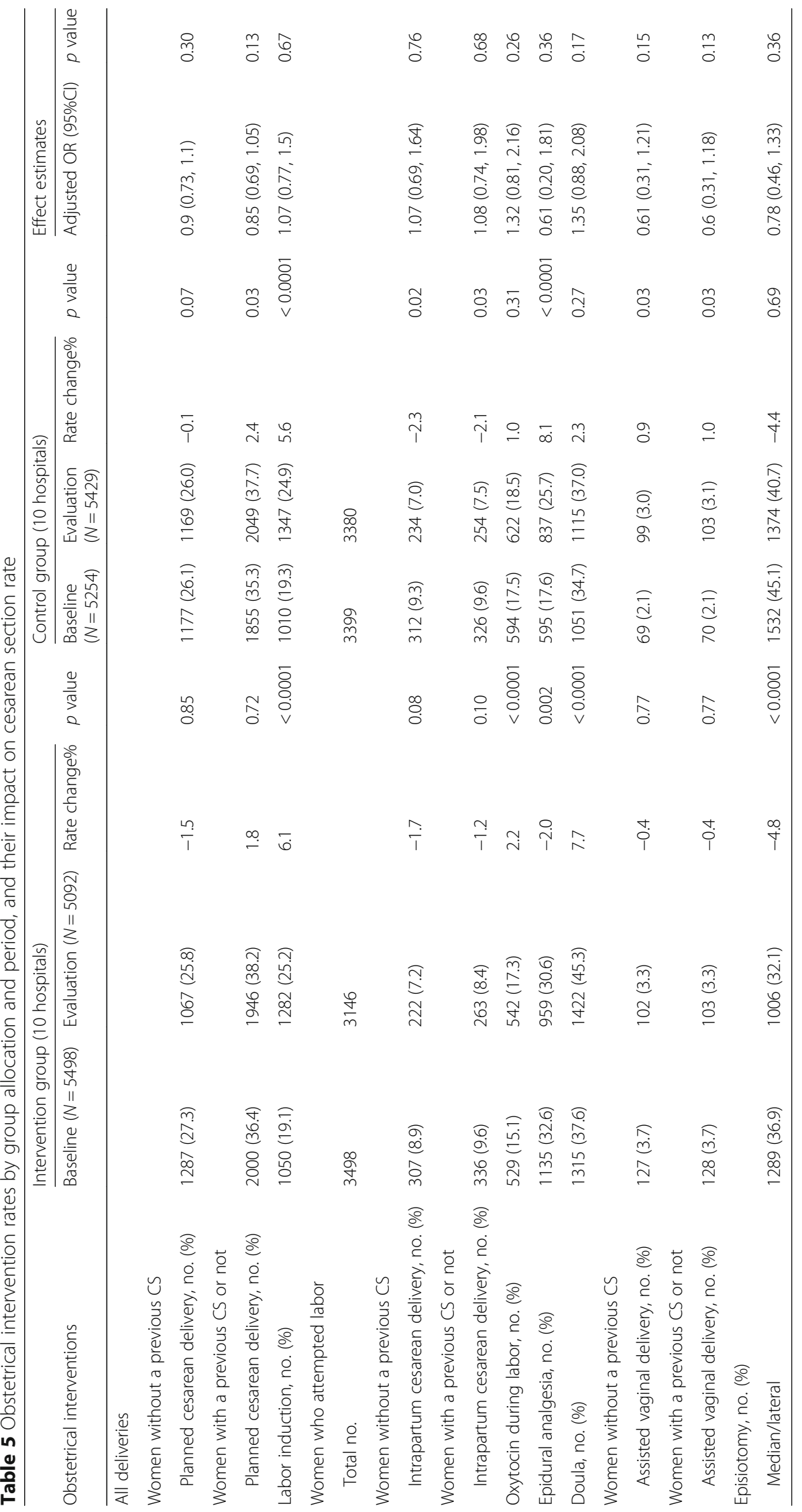


hospitals. Fourth, the high CS rate in Shanghai is mainly contributed by the high prelabor CS rate, which was our main target. Two of the three intervention components, namely health education and hospital CS policy, were designed to tackle this issue, particularly in nulliparous women. However, it takes time to change the culture and for the education to take effect. The duration of our intervention may be too short to see a significant impact. The impact might become statistically significant if the interventions continued for a longer period. Finally, our intervention package included three measures. It is difficult to disentangle their effects.

CS issue is extremely complex and deeply rooted. Despite our recognition of their importance, some measures were beyond what our study could do while other measures were difficult to implement. For example, we knew that fear of pain could be effectively addressed by providing epidural analgesia, but some hospitals were constrained by anesthesia resources. Only 8 hospitals in our study provided epidural analgesia, resulting in still low epidural analgesia use (20-30\% of women who attempted labor). Doula is effective in reducing CS use and increasing women's satisfaction. But the overwhelming volume of deliveries in Chinese public hospitals hampers one-to-one doula or midwife support. In our study, only one third of parturients had doula. And a doula is often shared by more than one laboring woman.

We also knew that physicians play a critical role in CS decision making, prelabor, and intrapartum. But the "physician factor" is also complicated by multiple forces. The shortage of medical staff to handle the large volume of delivery, financial incentives, and constrained doctor-patient relationship all likely draw the decision leaning towards CS. Physicians' practice pattern is often unclear. Our trial did not attempt to address the physician factor directly, which may be an important determinant in the success of an intervention trial on reducing the CS rate. For future studies, an assessment of practice pattern among physicians may provide useful insights. For example, asking the surgeon to complete a detailed reporting form that uncovers the indications as well as intentions and actions of the surgeon may help to identify potential targets for intervention. Given its importance, the physician factor could be a focus for future research. But the government health policy that can address some of the above issues may be more effective.

\section{Conclusions}

Our multifaceted intervention for 8 months was not effective in reducing the CS rate in a large, multicenter cluster-randomized field trial in Shanghai, China.
Further strategies that can be tailored to local contexts and drivers of CS are warranted to result in more effective measure to reduce the high CS rate. Government policy may have a greater impact on reducing the CS rate than the interventions initiated by hospitals or academic organizations.

\section{Supplementary information}

The online version of this article (https://doi.org/10.1186/s12916-020-1491-6) contains supplementary material, which is available to authorized users.

Additional file 1: Table S1. The list of health education classes available to pregnant women.

Additional file 2. Study protocol.

Additional file 3: Table S1. CONSORT 2010 checklist of information to include when reporting a cluster randomised trial. Table S2. Extension of CONSORT for abstracts 1,2 to reports of cluster randomised trials.

Additional file 4: Chart Abstraction Form for Maternal and Neonatal (or Stillbirth) Information.

\section{Abbreviations}

ACOG: American College of Obstetricians and Gynecologists; ART: Assisted reproductive technology; BMI: Body mass index; Cls: Confidence intervals; CS: Cesarean section; GA: Gestational age; GEE: Generalized estimating equations; GWG: Gestational weight gain; ORs: Odds ratios; QuIPS: Quality Improvement and Patient Safety Committee

\section{Acknowledgements}

This study was funded by the Shanghai Municipal Commission of Health and Family Planning (GWIV-26). The authors would like to thank the following hospitals and institutions for participating in this multicenter trial: Xinhua Hospital, Shanghai Jiao Tong University School of Medicine; Shanghai Maternal and Child Health Center; Shanghai Jiao Tong University School of Public Health; Minhang District Maternal and Child Health Hospital; Pudong New District Maternal and Child Health Hospital; Putuo District Maternal and Child Health Hospital; Pudong New Area People's Hospital; Eighth People's Hospital; Sixth People's Hospital, Shanghai Jiao Tong University School of Medicine; Jiading District Maternal and Child Health Hospital; First maternity and Infant Hospital, Tongji University School of Medicine; Songjiang District Maternal and Child Health Hospital; China Welfare Association International Peace Maternal and Child Health Hospital, Shanghai Jiao Tong University School of Medicine; Fudan University Obstetrics and Gynecology Hospital; Jinshan Hospital, Fudan University; Fifth People's Hospital, Fudan University; Minhang District Central Hospital; Changning District Maternal and Child Health Hospital; Putuo District Central Hospital; Zhujiajiao People's Hospital; Changhai Hospital, Second Military Medical University; Tongren Hospital, Shanghai Jiao Tong University School of Medicine.

\section{Authors' contributions}

$J Z, Z-L P, M Q, Z-L L, L Z$, and $M L$ contributed to the study design. JZ, Z-LP, MQ, L-GH, D-HY, RZ, Z-LL, ML, LZ, JX, Z-XH, M-ZN, YW, L-HP, L-XH, J-FH, L-YP, T-HJ, $\mathrm{Q}-\mathrm{XJ}, \mathrm{M}-\mathrm{YH}$, and $\mathrm{RH}$ contributed to participant recruitment and data collection. $J Z$, Z-LL, ML, LZ, and RH did the data analyses. Z-LL, ML, LZ, and JZ wrote the manuscript. All authors contributed to data interpretation, provided critical revision of the manuscript, approved the final manuscript, and agreed to submit for publication.

\section{Funding}

This study was funded by the Shanghai Municipal Commission of Health and Family Planning (GWIV-26). The funder of the study had no role in the study design, data collection, data analysis, data interpretation, or writing of the report. The corresponding author had full access to all the data in the study and had final responsibility for the decision to submit for publication.

\section{Availability of data and materials}

The datasets used and/or analyzed during the current study are available from the corresponding author on reasonable request. 


\section{Ethics approval and consent to participate}

An ethical approval was obtained from the Ethics Review Board of the Xinhua hospital, Shanghai Jiao Tong University School of Medicine and other participating hospitals. Conventional informed consent was not necessary in this study.

\section{Consent for publication}

Not applicable.

\section{Competing interests}

The authors declare that they have no competing interests.

\section{Author details}

'Ministry of Education-Shanghai Key Laboratory of Children's Environmental Health, Xinhua Hospital, Shanghai Jiao Tong University School of Medicine, Shanghai, China. ${ }^{2}$ Department of Obstetrics and Gynecology, Xinhua Hospital, Shanghai Jiao Tong University School of Medicine, Shanghai, China. ${ }^{3}$ Shanghai Jiao Tong University School of Public Health, Shanghai, China. ${ }^{4}$ Department of Obstetrics, Jiading District Maternal and Child Health Hospital, Shanghai, China. ${ }^{5}$ Department of Maternal Health Care, Minhang District Maternal and Child Health Hospital, Shanghai, China. ${ }^{6}$ Department of Obstetrics, First Maternity and Infant Hospital, Tongji University School of Medicine, Shanghai, China. 'Department of Obstetrics, Songjiang District Maternal and Child Health Hospital, Shanghai, China. ${ }^{8}$ Department of Obstetrics and Gynecology, Sixth People's Hospital, Shanghai Jiao Tong University School of Medicine, Shanghai, China. ${ }^{9}$ Department of Obstetrics, China Welfare Association International Peace Maternal and Child Health Hospital, Shanghai Jiao Tong University School of Medicine, Shanghai, China. ${ }^{10}$ Department of Obstetrics and Gynecology, Pudong New District Maternal and Child Health Hospital, Shanghai, China. "'Department of Obstetrics and Gynecology, Pudong New Area People's Hospital, Shanghai, China.

${ }^{12}$ Department of Obstetrics, Putuo District Maternal and Child Health Hospital, Shanghai, China. ${ }^{13}$ Department of General Surgery, Eighth People's Hospital, Shanghai, China. ${ }^{14}$ Center for HTA, China Hospital Development Institute, Shanghai Jiao Tong University, Shanghai, China. ${ }^{15}$ Nursing College, Shanghai University of Medicine \& Health Sciences, Shanghai, China.

${ }^{16}$ Shanghai Maternal and Child Health Center, Shanghai, China.

\section{Received: 12 September 2019 Accepted: 7 January 2020}

Published online: 14 February 2020

\section{References}

1. Boerma T, Ronsmans C, Melesse DY, Barros AJD, Barros FC, Juan L, et al. Global epidemiology of use of and disparities in caesarean sections. Lancet. 2018;392:1341-8.

2. Li HT, Luo S, Trasande L, Hellerstein S, Kang C, Li JX, et al. Geographic variations and temporal trends in cesarean delivery rates in China, 20082014. JAMA. 2017;317:69-76.

3. Mi J, Liu F. Rate of caesarean section is alarming in China. Lancet. 2014;383: 1463-4.

4. Long Q, Kingdon C, Yang F, Renecle MD, Jahanfar S, Bohren MA, et al. Prevalence of and reasons for women's, family members', and health professionals' preferences for cesarean section in China: a mixed-methods systematic review. PLoS Med. 2018;15:e1002672.

5. Hellerstein S, Feldman S, Duan T. China's 50\% caesarean delivery rate: is it too high? BJOG. 2015;122:160-4.

6. Betrán AP, Temmerman M, Kingdon C, Mohiddin A, Opiyo N, Torloni MR, et al. Interventions to reduce unnecessary caesarean sections in healthy women and babies. Lancet. 2018;392:1358-68.

7. Jiang $Y$, Ying $X$, Kane S, Mukhopadhyay M, Qian X. Violence against doctors in China. Lancet. 2014;384:744-5.

8. Sandall J, Tribe RM, Avery L, Mola G, Visser GHA, Homer CSE, et al. Shortterm and long-term effects of caesarean section on the health of women and children. Lancet. 2018;392:1349-57.

9. Keag OE, Norman JE, Stock SJ. Long-term risks and benefits associated with cesarean delivery for mother, baby, and subsequent pregnancies: systematic review and meta-analysis. PLoS Med. 2018;15:e1002494.

10. Johri M, Ng ESW, Bermudez-Tamayo C, Hoch JS, Ducruet T, Chaillet N. A cluster-randomized trial to reduce caesarean delivery rates in Quebec: costeffectiveness analysis. BMC Med. 2017;15:96.
11. Yuqi $L$, Tan $G$, Chengming $S$, Xuri $S$. The ICU is becoming a main battlefield for severe maternal rescue in China: an 8-year single-center clinical experience. Crit Care Med. 2017;45:e1106-e10.

12. Liang J, Mu Y, Li X, Tang W, Wang Y, Liu Z, et al. Relaxation of the one child policy and trends in caesarean section rates and birth outcomes in China between 2012 and 2016: observational study of nearly seven million health facility births. BMJ. 2018;360:k817.

13. Hellerstein S, Feldman S, Duan T. Survey of obstetric care and cesarean delivery rates in Shanghai, China. Birth. 2016;43:193-9.

14. Chen Y. Maternal age and cesarean delivery rate in Shanghai. Am J Public Health. 1993;83:287-8.

15. J Z, H C, Z Z, X Z, XX, L Z, et al. Investigation and analysis of cesarean section rate and indications in Shanghai. Chinese Journal of Practical Gynecology and Obstetrics. 2019;35:325-29.

16. Khunpradit S, Tavender E, Lumbiganon P, Laopaiboon M, Wasiak J, Gruen RL. Non-clinical interventions for reducing unnecessary caesarean section. Cochrane Database Syst Rev. 2011;15:Cd005528.

17. Ji H, Jiang H, Yang L, Qian X, Tang S. Factors contributing to the rapid rise of caesarean section: a prospective study of primiparous Chinese women in Shanghai. BMJ Open. 2015;5:e008994.

18. Long $Q$, Klemetti $R$, Wang $Y$, Tao F, Yan H, Hemminki E. High caesarean section rate in rural China: is it related to health insurance (new cooperative medical scheme)? Soc Sci Med. 2012;75:733-7.

19. Portela MC, Pronovost PJ, Woodcock T, Carter P, Dixon-Woods M. How to study improvement interventions: a brief overview of possible study types. BMJ Qual Saf. 2015;24:325-36.

20. Kingdon C, Downe S, Betran AP. Non-clinical interventions to reduce unnecessary caesarean section targeted at organisations, facilities and systems: systematic review of qualitative studies. PLoS One. 2018;13: e0203274.

21. Kingdon C, Downe S, Betran AP. Women's and communities' views of targeted educational interventions to reduce unnecessary caesarean section: a qualitative evidence synthesis. Reprod Health. 2018;15:130.

22. Chaillet N, Dumont A. Evidence-based strategies for reducing cesarean section rates: a meta-analysis. Birth. 2007;34:53-64.

23. World Health Organization. WHO recommendations non-clinical interventions to reduce unnecessary caesarean sections. Geneva: World Health Organization; 2018.

24. Marshall JL, Spiby H, McCormick F. Evaluating the 'Focus on Normal Birth and Reducing Caesarean section Rates Rapid Improvement Programme': a mixed method study in England. Midwifery. 2015;31:332-40.

25. Cai WW, Marks JS, Chen CH, Zhuang YX, Morris L, Harris JR. Increased cesarean section rates and emerging patterns of health insurance in Shanghai, China. Am J Public Health. 1998;88:777-80.

26. Ming Y, Li M, Dai F, Huang R, Zhang J, Zhang L, et al. Dissecting the current caesarean section rate in Shanghai, China. Sci Rep. 2019;9:2080.

27. Althabe F, Belizan JM, Villar J, Alexander S, Bergel E, Ramos S, et al. Mandatory second opinion to reduce rates of unnecessary caesarean sections in Latin America: a cluster randomised controlled trial. Lancet. 2004; 363:1934-40.

28. Liang WH, Yuan CC, Hung JH, Yang ML, Yang MJ, Chen YJ, et al. Effect of peer review and trial of labor on lowering cesarean section rates. J Chin Med Assoc. 2004:67:281-6.

29. Chen CS, Liu TC, Chen B, Lin CL. The failure of financial incentive? The seemingly inexorable rise of cesarean section. Soc Sci Med. 2014;101:47-51.

30. Chung SH, Seol HJ, Choi YS, Oh SY, Kim A, Bae CW. Changes in the cesarean section rate in Korea (1982-2012) and a review of the associated factors. J Korean Med Sci. 2014;29:1341-52.

31. Walker R, Turnbull D, Wilkinson C. Strategies to address global cesarean section rates: a review of the evidence. Birth. 2002;29:28-39.

32. Mann S, Pratt S, Gluck P, Nielsen P, Risser D, Greenberg P, et al. Assessing quality obstetrical care: development of standardized measures. Jt Comm J Qual Patient Saf. 2006;32:497-505.

33. Zeger SL, Liang KY, Albert PS. Models for longitudinal data: a generalized estimating equation approach. Biometrics. 1988;44:1049-60.

34. Chaillet N, Dumont A, Abrahamowicz M, Pasquier JC, Audibert F, Monnier P, et al. A cluster-randomized trial to reduce cesarean delivery rates in Quebec. N Engl J Med. 2015:372:1710-21.

35. Zhang J, Geerts C, Hukkelhoven C, Offerhaus P, Zwart J, de Jonge A. Caesarean section rates in subgroups of women and perinatal outcomes. BJOG. 2016;123:754-61. 
36. Runmei $\mathrm{M}$, Terence TL, Yonghu S, Hong X, Yuqin T, Bailuan L, et al. Practice audits to reduce caesareans in a tertiary referral hospital in South-Western China. Bull World Health Org. 2012;90:488-94.

37. Brown HC, Paranjothy S, Dowswell T, Thomas J. Package of care for active management in labour for reducing caesarean section rates in low-risk women. Cochrane Database Syst Rev. 2013;9:Cd004907.

38. Ayres-De-Campos D, Cruz J, Medeiros-Borges C, Costa-Santos C, Vicente L. Lowered national cesarean section rates after a concerted action. Acta Obstet Gynecol Scand. 2015;94:391-8

39. Chen I, Opiyo N, Tavender E, Mortazhejri S, Rader T, Petkovic J, et al. Nonclinical interventions for reducing unnecessary caesarean section. Cochrane Database Syst Rev. 2018;9:Cd005528.

40. Yu Y, Zhang X, Sun C, Zhou H, Zhang Q, Chen C. Reducing the rate of cesarean delivery on maternal request through institutional and policy interventions in Wenzhou, China. PLoS One. 2017:12:e0186304.

41. Liu X, Lynch CD, Cheng WW, Landon MB. Lowering the high rate of caesarean delivery in China: an experience from Shanghai. BJOG. 2016;123: $1620-8$.

42. Poma PA. Effect of departmental policies on cesarean delivery rates: a community hospital experience. Obstet Gynaecol. 1998;91:1013-8.

43. Lagrew DC Jr, Morgan MA. Decreasing the cesarean section rate in a private hospital: success without mandated clinical changes. Am J Obstet Gynecol. 1996;174:184-91.

44. Lumbiganon P, Laopaiboon M, Gulmezoglu AM, Souza JP, Taneepanichskul S, Ruyan P, et al. Method of delivery and pregnancy outcomes in Asia: the WHO global survey on maternal and perinatal health 2007-08. Lancet. 2010; 375:490-9.

45. Kingdon C, Downe S, Betran AP. Interventions targeted at health professionals to reduce unnecessary caesarean sections: a qualitative evidence synthesis. BMJ Open. 2018;8:e025073.

46. Yao J, Zhu X, Lu H. Assessing the midwifery workforce demand: Utilising birthrate plus in China. Midwifery. 2016;42:61-6.

\section{Publisher's Note}

Springer Nature remains neutral with regard to jurisdictional claims in published maps and institutional affiliations.

Ready to submit your research? Choose BMC and benefit from:

- fast, convenient online submission

- thorough peer review by experienced researchers in your field

- rapid publication on acceptance

- support for research data, including large and complex data types

- gold Open Access which fosters wider collaboration and increased citations

- maximum visibility for your research: over $100 \mathrm{M}$ website views per year

At $\mathrm{BMC}$, research is always in progress.

Learn more biomedcentral.com/submissions 